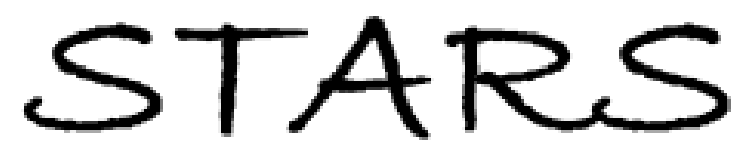

University of Central Florida

STARS

$1-1-2006$

\title{
Interfacial phonon scattering in semiconductor nanowires by molecular-dynamics simulation
}

\author{
Brian Becker \\ University of Central Florida \\ Patrick K. Schelling \\ University of Central Florida \\ Simon R. Phillpot
}

Find similar works at: https://stars.library.ucf.edu/facultybib2000 University of Central Florida Libraries http://library.ucf.edu

This Article is brought to you for free and open access by the Faculty Bibliography at STARS. It has been accepted for inclusion in Faculty Bibliography 2000 s by an authorized administrator of STARS. For more information, please contact STARS@ucf.edu.

\section{Recommended Citation}

Becker, Brian; Schelling, Patrick K.; and Phillpot, Simon R., "Interfacial phonon scattering in semiconductor nanowires by molecular-dynamics simulation" (2006). Faculty Bibliography 2000s. 5932.

https://stars.library.ucf.edu/facultybib2000/5932

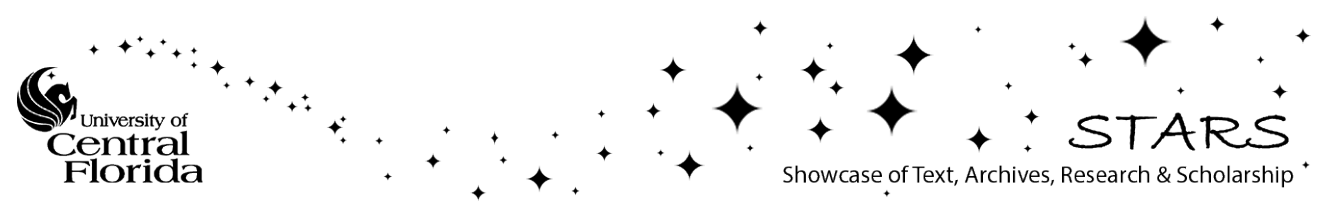




\section{Interfacial phonon scattering in semiconductor nanowires by molecular- dynamics simulation}

Cite as: J. Appl. Phys. 99, 123715 (2006); https://doi.org/10.1063/1.2207503

Submitted: 04 October 2005 . Accepted: 05 April 2006 . Published Online: 28 June 2006

Brian Becker, Patrick K. Schelling, and Simon R. Phillpot

ARTICLES YOU MAY BE INTERESTED IN

Phonon wave-packet dynamics at semiconductor interfaces by molecular-dynamics simulation

Applied Physics Letters 80, 2484 (2002); https://doi.org/10.1063/1.1465106

Kapitza conductance and phonon scattering at grain boundaries by simulation

Journal of Applied Physics 95, 6082 (2004); https://doi.org/10.1063/1.1702100

Nanoscale thermal transport

Journal of Applied Physics 93, 793 (2003); https://doi.org/10.1063/1.1524305

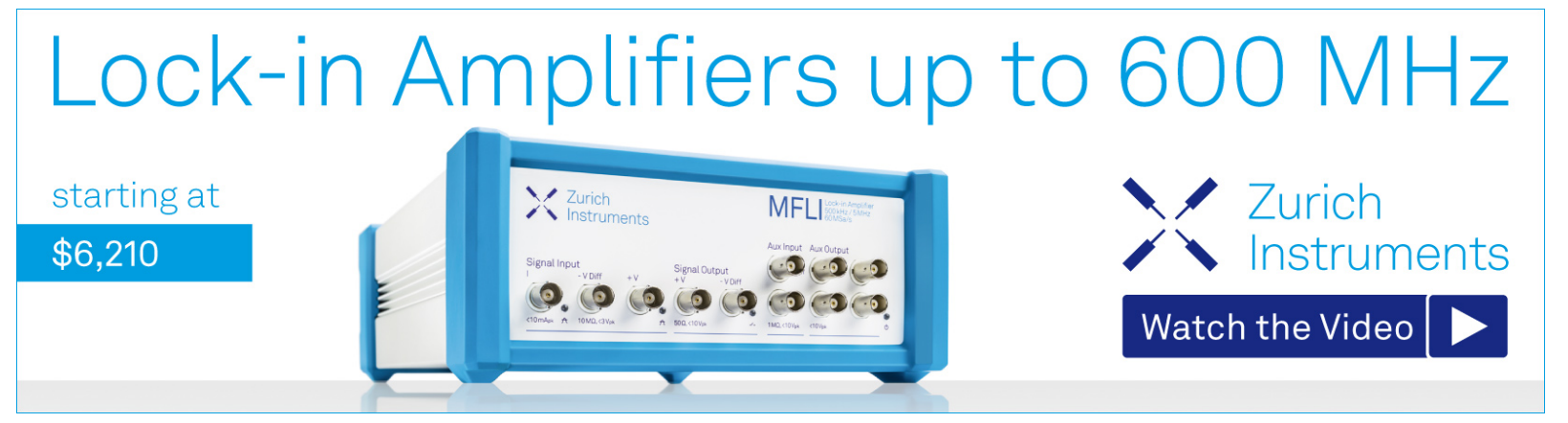

J. Appl. Phys. 99, 123715 (2006); https://doi.org/10.1063/1.2207503 


\title{
Interfacial phonon scattering in semiconductor nanowires by molecular-dynamics simulation
}

\author{
Brian Becker and Patrick K. Schelling ${ }^{\text {a) }}$ \\ Advanced Materials Processing and Analysis Center, University of Central Florida, Orlando, \\ Florida 32816 and Department of Physics, University of Central Florida, Orlando, Florida 32816 \\ Simon R. Phillpot \\ Department of Materials Science and Engineering, University of Florida, Gainesville, Florida 32611
}

(Received 4 October 2005; accepted 5 April 2006; published online 28 June 2006)

\begin{abstract}
We use molecular-dynamics simulations of vibrational wave packets to study the scattering of longitudinal-acoustic modes from interfaces in semiconductor nanowires of varying diameters. The energy transmission coefficient at the interface is found to depend strongly on both the nanowire diameter and the frequency of the incident wave. By analyzing the scattering events, we determine the selection rules for nanowire scattering that can be understood in terms of the representations of the point-group symmetry of the nanowire. Using such symmetry arguments, we predict that the presence of gaps in the phonon spectrum of thin high-symmetry nanowires will result in a complete reflection of phonons at the interfaces. We discuss the implications of our results for interfacial scattering in real systems, including $\mathrm{Si} / \mathrm{Ge}$ superlattice nanowires. (C) 2006 American Institute of Physics. [DOI: 10.1063/1.2207503]
\end{abstract}

\section{INTRODUCTION}

Semiconductor nanowires are becoming increasingly important in a variety of applications, including application in quantum wire lasers, ${ }^{1}$ field-effect transistors, ${ }^{2}$ and thermoelectric devices. ${ }^{3,4}$ These applications have spurred research into a variety of physical properties, including their thermaltransport behavior. It has now been established that nanowires exhibit thermal conductivities that are considerably lower than those of bulk materials of the same composition. For example, silicon nanowires with diameters less than $100 \mathrm{~nm}$ show thermal conductivities that are as much as two orders of magnitude lower than those of bulk silicon. ${ }^{5}$ These results suggest that nanowires may hold promise as thermoelectrics, where the goal is to reduce the thermal conductivity, thereby increasing the thermoelectric figure of merit. ${ }^{3,4,6}$ In contrast to this considerable promise for thermoelectrics, the very low thermal conductivities of nanowires suggest that their application to lasers and transistors may be limited. Regardless of the specific application, however, it is clear that the systematic development of thermal-transport applications of nanowires will be greatly accelerated by a detailed understanding of the material parameters that determine thermal-transport properties and by the ability to engineer them.

Although our theoretical understanding of thermal transport at the nanoscale is still in its infancy, ${ }^{7}$ a consensus is emerging from a variety of theoretical approaches that surface scattering is responsible for the low thermal conductivity of nanowires. ${ }^{8-12}$ In particular, the strong size dependence of the thermal conductivity of relatively thick silicon nanowires (with diameter $d>30 \mathrm{~nm}$ ) has been interpreted to be a result of both a strong surface scattering and a size-

${ }^{a)}$ Electronic mail: pschell@mail.ucf.edu dependent phonon density of states (DOS). ${ }^{8}$ For the work in Ref. 8, the authors used realistic calculations of the phonon dispersions with bulk expressions for the scattering rates. In the limit of very small diameters (below $30 \mathrm{~nm}$ ), however, this approach fails to even qualitatively describe the experimental data. ${ }^{5,8}$ This suggests that there may be a regime at very small sizes where scattering, either by defects, interfaces, or from anharmonic phonon-phonon interactions, is quite different than in the bulk and that theoretical models are required.

In this article we use atomic-level simulation techniques to systematically explore phonon transport in small diameter (below $3 \mathrm{~nm}$ ) nanowires and to identify the fundamental principles by which the phonon transport in such systems can be understood. The tool we use is molecular-dynamics (MD) simulation, in which the full spectrum of vibrational excitations of the system can be described. In the specific MD approach taken here, the scattering of individual, coherent wave packets from an interface is characterized, from which a detailed mechanistic understanding of the scattering process is obtained. We find that the phonon transmission coefficient is strongly and nonmonotonically dependent on frequency, with a dependence that is directly linked to the presence of surfaces. The results are interpreted based on the underlying symmetry of the nanowire, leading to the identification of general selection rules that apply to the case of scattering at interfaces in a nanowire. We also view these silicon nanowires as a laboratory in which to elucidate phonon-scattering mechanism of general relevance to nanowire systems.

\section{METHODOLOGY}

The use of phonon wave packets for the study of phonon interfacial scattering is described in detail elsewhere ${ }^{13}$ here we provide only a brief outline of the most pertinent details. 


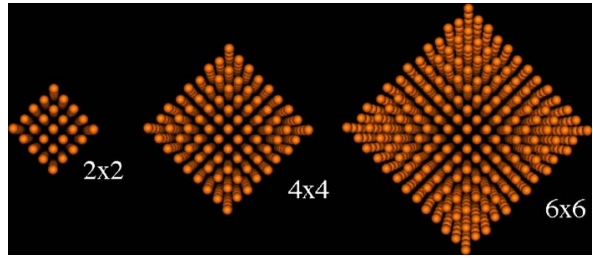

FIG. 1. Illustration of an atomic lattice structure of (110) surfaces for nanowires made from $2 \times 2,4 \times 4$, and $6 \times 6$ unit cells for the diameter. (110) surfaces have more stable triply coordinated surface atoms.

The basic idea is to construct a linear combination of normal modes within a particular phonon branch that results in a spatially localized wave packet. For an infinite, periodic crystal, these normal modes are Bloch states. For a nanowire these states are also Bloch states, but propagation is only allowed along the axis of the nanowire. Assuming that propagation can only occur in one dimension, the initial atomic displacements are given by

$$
u_{j l \mu}(0)=\sum_{k} a_{\lambda k} \varepsilon_{j \mu \lambda k} \exp \left(i k z_{l}\right) \text {. }
$$

Here, $u_{j l \mu}(0)$ represents the $\mu$ th Cartesian component of the displacement for atom $j$ in the unit cell labeled $l$, and the coordinate of the unit cell $l$ is given by $z_{l}$. The polarization vector $\varepsilon_{j \mu \lambda k}$ of the normal mode with wave number $k$ in the band labeled $\lambda$ is found by diagonalizing the dynamical matrix of the system under consideration. In this case, as described below, the dynamical matrix characterizes a nanowire structure. For a confined nanowire structure, the propagation is one dimensional, and hence the wave vector $k$ has only one component. The coefficients $a_{\lambda k}$, chosen to result in a spatially localized wave packet, are determined by a method described in our previous work. ${ }^{13}$ The result is a wave packet localized in real and $k$ spaces that corresponds to a packet of phonons within a single phonon branch $\lambda$. The propagation of the wave packet is accomplished by MD simulation, which for small amplitudes of the modes $a_{\lambda k}$ does not result in any significant phonon-phonon scattering in a perfect lattice. When an interface or defect is introduced, or if the amplitude of the wave packet is sufficiently high that the harmonic approximation is no longer valid, scattering will occur and the results can be studied in detail. The capabilities of this approach have been demonstrated in the context of studies of simple epitaxial interfaces, ${ }^{13}$ including a $\mathrm{Si} / \mathrm{Ge}$ interface, ${ }^{14}$ superlattices, ${ }^{15}$ grain boundaries, ${ }^{16}$ and optical-phonon hot spots in silicon. ${ }^{17}$

The nanowire structures studied are shown in Fig. 1. In each case, the nanowire axis lies along the [001] crystal axis of the silicon diamond lattice. The surfaces are each (110) type. This particular choice was made because preliminary studies showed that nanowires with some other surfaces were unstable during a MD simulation. In particular, nanowires with (100)-type surfaces, which are quite high energy when not reconstructed, were found to become amorphous under MD annealing. Attempts to build a reconstruction of the nanowire surface with (100)-type surfaces, based on the well-known $2 \times 1$ (100) surface reconstruction, ${ }^{18,19}$ were also unsuccessful. The (110) surface, on the other hand, has three-

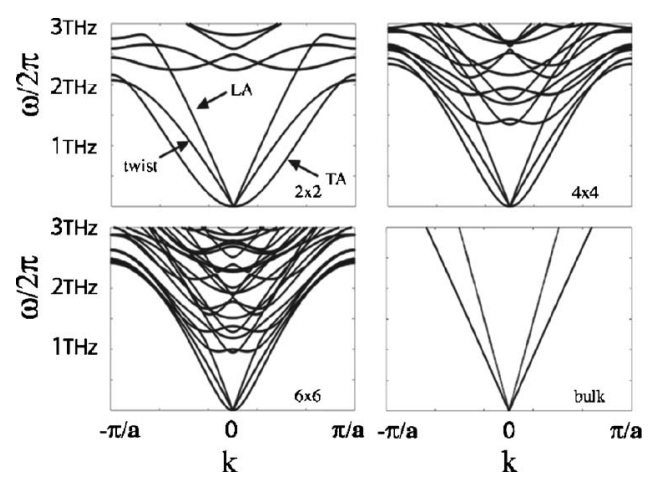

FIG. 2. Phonon dispersion relations computed for the nanowire structures whose cross sections are shown in Fig. 1. Only the low-frequency portion of the spectrum relevant to the study here is shown. The full spectrum extends in each case up to about $18 \mathrm{THz}$. Also shown for comparison are the phonon dispersions for bulk silicon along [001].

fold coordinated surface atoms and is lower in energy than the unreconstructed (100) surface, and nanowires made with this surface termination were found to be stable with respect to disordering or surface reconstructions up to MD simulation temperatures of $500 \mathrm{~K}$ and perhaps beyond. The structures shown in Fig. 1 differ from each other only in their diameter and, hence, the number of atoms in the structure that periodically repeats along the $z$ axis. In particular, the smallest wire has 25 atoms per repeat unit and a diameter of about $0.76 \mathrm{~nm}$. This repeat unit of the structure was generated starting from a square section of the diamond lattice with two nonprimitive unit cells on a side. This was then cut along (110)-type planes to create the (110)-type surfaces. Henceforth, we will refer to this as the $2 \times 2$ nanowire. The intermediate size wire has 81 atoms per repeat unit and a diameter of about $1.52 \mathrm{~nm}$. Finally, the largest wire has 169 atoms in the repeat unit and a diameter of $2.28 \mathrm{~nm}$. These latter two nanowires will be hereafter referred to as the 4 $\times 4$ and $6 \times 6$ nanowires, respectively.

The interatomic interactions were described by the wellknown Stillinger-Weber (SW) potential for silicon. ${ }^{20}$ Due to the short-ranged interactions in the SW model, as long as atoms are in perfect diamond lattice positions at the bulk lattice constant of silicon $a_{0}=5.43 \AA$, there are no forces on the atoms even when there is a surface. As a result, the atoms in the structures shown in Fig. 1 do not exhibit surface relaxation, as one would expect in a real system. Furthermore, there is no reconstruction of our surface under simulated annealing; this is in contrast to experiment, where the (110) is known to exhibit various reconstructions. ${ }^{21}$ Since our conclusions are based on an understanding of the particular symmetry of the nanowire, though not on the details of the structure, they are not vitiated by the absence of surface relaxations or reconstructions.

Figure 2 shows the calculated phonon dispersions in the low-frequency regime for the nanowire structures in Fig. 1. Although not shown, the spectra extend to maximum frequencies comparable to that observed in bulk silicon, with the observed maxima for the nanowires at approximately 17.0 THz independent of the nanowire diameter. In contrast to a bulk system that has three acoustic branches, the nanowire has, in addition, a torsional acoustic branch that corre- 
sponds to the twisting of the nanowire. This mode is not present in the bulk system because torsional modes are inconsistent with the assertion that the phonon modes are Bloch states. In addition, because the atoms at the surfaces are undercoordinated, the restoring forces for compression and bending are less than they would be in bulk silicon, and hence the nanowire shows sharply decreased longitudinalacoustic (LA) and transverse-acoustic (TA) group velocities compared to bulk silicon. This can be seen immediately by considering the phonon frequencies at the zone-edge phonon frequencies. For example, the LA modes at the zone edge in our calculation are just less than $3.0 \mathrm{THz}$. By contrast, the zone-edge LA modes in bulk silicon have a frequency of about $13.0 \mathrm{THz}$, though the Brillouin zone for the bulk extends twice as far in the $k$ space as the Brillouin zone of the nanowire. Because of the high symmetry of our nanowire structures (see Fig. 1), the TA modes are degenerate; a more detailed discussion of the effects of symmetry follows in the next section. Interestingly, the flexural or TA modes in the nanowire display a quadratic dependence on $k$; this behavior was previously also seen in carbon nanotubes ${ }^{22}$ and silicon nanowires. ${ }^{23}$ Finally, the frequency of the lowest "optical" modes (i.e., those modes that have nonzero frequency at $k$ $=0$ ) depends strongly on the diameter of the nanowire; in particular, as the diameter of the nanowire increases, the number of low-frequency optical modes also increases. This behavior is analogous to waveguides in electromagnetism, where the maximum allowed wavelength perpendicular to the propagation direction increases as the lateral dimensions increase.

The phonon spectra for silicon nanowires have previously been computed by other authors using classical interatomic interactions; in particular, Mingo has computed the spectrum of silicon nanowires using a harmonic potential. ${ }^{24}$ Our results are rather comparable; for example, the calculated zone-edge acoustic frequencies of about 3-4 THz are similar to our calculated value of just below $3 \mathrm{THz}$. The SW potential was also used in Ref. 23 to compute the phonon spectra for a silicon nanowire, but with the growth direction of the nanowire along [111]. While there are similar features in our spectra to that of Ref. 23, there are also some notable differences. For example, because of the particular symmetry of the nanowires considered in this article, the flexural modes (i.e., the TA modes) are degenerate, whereas the nanowire studied in Ref. 23 was of lower symmetry, and hence the flexural modes were not degenerate.

To create a simple interface that will scatter phonons we consider, as in our previous work, ${ }^{13}$ an interface formed from two nanowire segments that are joined at a perfectly coherent interface (see Fig. 3). The only difference between the two segments is in the masses of the atoms. In particular, the phonon wave packets are introduced in material $A$, whose atoms have the mass of silicon, $M_{\mathrm{Si}}=28 \mathrm{amu}$, and are allowed to propagate and scatter from material $B$ whose atoms have mass $4 M_{\mathrm{Si}}$. Because the two materials only differ in their atomic masses, the phonon dispersion curves are identical, except that the phonon frequencies of material $B$ are lower by a factor of 2 . In all of our simulations, the amplitude of the atomic displacements that make up the phonon

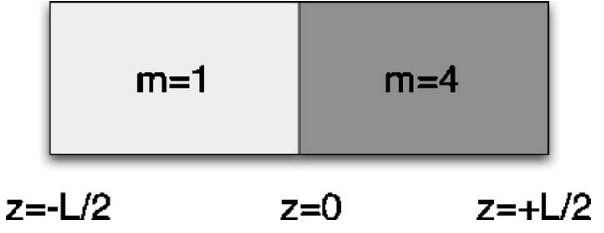

FIG. 3. Schematic of the nanowire structure with the mass discontinuity. For atoms with $z<0$ (material $A$ ), the atoms have mass $M_{\mathrm{Si}}(28 \mathrm{amu})$. For atoms with $z>0$ (material $B$ ), the atoms have mass $4 M_{\mathrm{Si}}$. The phonon wave packets are introduced in material $A$ and allowed to propagate towards the interface at $z=0$ where they scatter from the heavier atoms in material $B$. The total length of the simulation cell was 1000 units cells $(543 \mathrm{~nm})$.

wave packet is quite low (below $10^{-4} a_{0}$ ), with the rest of the lattice being initially at zero temperature. As a result, the scattering is harmonic, and we expect that the frequencies of the scattered waves will be identical to that of the incident LA wave packet. Finally, by analyzing the atomic displacements after the scattering event, detailed mechanistic information can be obtained about scattering in the nanowire. This analysis is described in the next section.

\section{MD RESULTS AND ANALYSIS FOR INTERFACIAL WAVE PACKET SCATTERING}

The quantity most relevant to understanding the interfacial thermal conductance (i.e., the Kapitza conductance) is the energy transmission coefficient $\alpha$, the ratio of the energy transmitted across the interface to the energy of the incident wave packet. In other words, if we compute the energy of the incident wave packet to be $\varepsilon_{\text {inc }}$ and the energy of the transmitted waves to be $\varepsilon_{\text {trans }}$, the energy transmission coefficient is $\alpha=\varepsilon_{\text {trans }} / \varepsilon_{\text {inc }}$. As described in the previous section, the wave packets are formed from linear combinations of the eigenstates of the normal modes of a nanowire of material $A$, and hence they propagate without scattering until they encounter the interface with material $B$. This is shown in a series of snapshots in Fig. 4. In Fig. 4, an incident LA wave packet with $k=0.080 \pi / a_{0}$ and an average frequency of $0.36 \mathrm{THz}$ propagates without scattering in material $A$. When

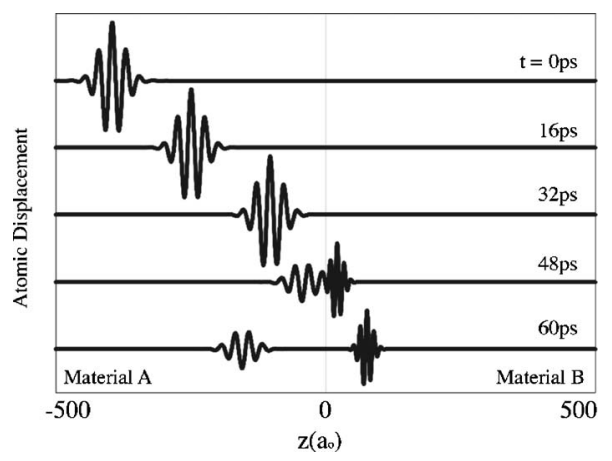

FIG. 4. Series of snapshots showing propagation and scattering of a LA wave packet introduced in the $2 \times 2$ nanowire of material $A$. The wave packet is constructed from the normal modes of the $2 \times 2$ nanowire and hence does not scatter until the interface with material $B$ is encountered. After scattering, part of the incident wave is reflected back into material $A$ as a LA mode, but propagating away from the interface. The transmitted mode in material $B$ in this case is also a LA mode, but with a shorter wavelength than the incident wave. 


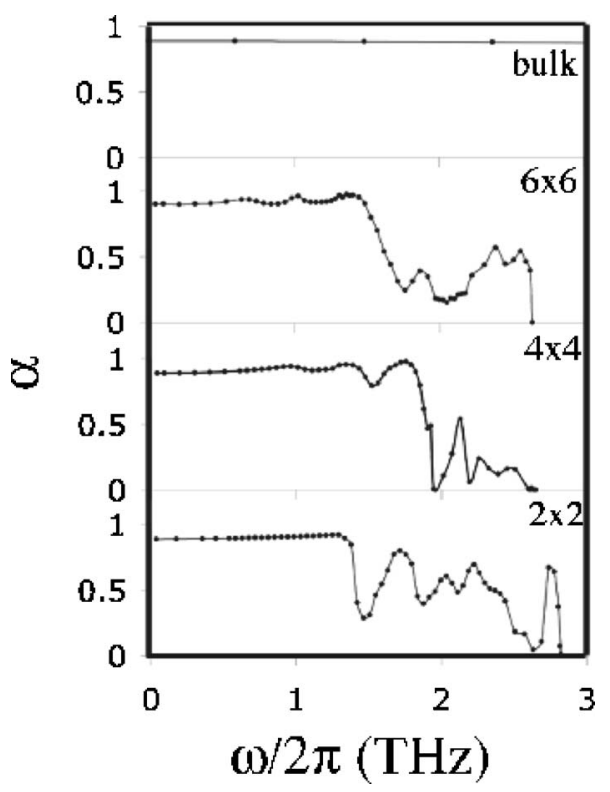

FIG. 5. Energy transmission coefficient $\alpha$ as a function of the frequency of the incident LA wave packet for the $2 \times 2,4 \times 4$, and $6 \times 6$ nanowires. For comparison, we also include results from scattering in a bulk system from Ref. 8. For long wavelengths, the MD results in each case are consistent with the prediction of $8 / 9$ from the AMM. The transmission coefficient drops abruptly near $1.4 \mathrm{THz}$ when the incident frequency is greater than the LA frequency for the atoms in the $z>0$ region. Above this cutoff, energy is transmitted into optical-acoustic bands.

the wave packet encounters the interface, it is scattered into transmitted waves in material $B$ and waves reflected back into material $A$.

Figure 5 shows the energy transmission coefficient for incident LA wave packets for each of the wires, determined from a series of simulations of the scattering of phonon wave packets from the interfaces. For comparison, we also include our previous results for a bulk system with the same mass discontinuity. ${ }^{13}$

In the long wavelength limit, the transmission coefficient for each system tends toward the limit of $\alpha=8 / 9$, predicted from the acoustic-mismatch model (AMM) ${ }^{13,25}$ At somewhat higher frequencies (i.e., shorter wavelengths), the continuum approximation of the AMM breaks down. In particular, $\alpha$ exhibits a sharp decrease near a frequency of $\omega / 2 \pi$ $\sim 1.4 \mathrm{THz}$. Referring to the vibrational spectra for material $A$ shown in Fig. 2, we see that the highest frequency for LA modes is near $2.8 \mathrm{THz}$. There is some dependence of this frequency on the nanowire diameter, and to be more precise the maximum LA mode frequencies are 2.82, 2.65, and $2.63 \mathrm{THz}$ for the $2 \times 2,4 \times 4$, and $6 \times 6$ nanowires, respectively. This means that the highest frequency of the LA modes in material $B$ is near 1.3-1.4 THz. Hence, the sharp decrease in $\alpha$ near $1.4 \mathrm{THz}$ corresponds to the maximum frequency of a LA mode in material $B$, above which LA $\rightarrow$ LA transmission is not allowed.

In the high-frequency regime (i.e., above the cutoff for LA $\rightarrow$ LA transmission), although the transmission coefficient drops off dramatically, a significant fraction of the incident energy is still transmitted into material $B$. This is scattering from LA to "optical" acoustic (OA) modes. We determine the distribution of modes into which the energy is scattered by analyzing the final positions and velocities of the atoms long after the wave packet has interacted with the interface. In particular, we find the amplitudes of the normal modes $a_{\lambda k}$ from

$$
a_{\lambda k}=\sum_{j l \mu}\left[u_{i l \mu}\left(t_{f}\right)-\frac{i v_{j l \mu}\left(t_{f}\right)}{\omega_{\lambda k}}\right] \varepsilon^{*}{ }_{i \mu \lambda k} \exp \left(-i k z_{l}\right),
$$

where $u_{i l \mu}\left(t_{f}\right)$ and $v_{j l \mu}\left(t_{f}\right)$ are, respectively, the final displacements and velocities of the atoms at the final time $t_{f}$, and $\omega_{\lambda k}$ is the frequency of the normal modes. This approach is similar to that in our previous work, although in this case we include a velocity term in Eq. (2) and use the actual Bloch states, whereas in Ref. 16 we used a basis of standing waves. The advantage of Eq. (2) is that we can determine directly whether $k$ is positive or negative, whereas previously when working with a basis of standing waves, this distinction could not be made. Having determined the expansion coefficients $a_{\lambda k}$ from Eq. (2), the energy $\varepsilon_{\lambda k}$ in each mode $\lambda, k$ can then be found from

$$
\varepsilon_{\lambda k}=M\left|a_{\lambda k}\right|^{2} \omega_{\lambda k}^{2},
$$

where $M$ is the total mass of either material $A$ or material $B$.

We have analyzed the amount of energy transmitted and reflected into the various modes. As expected, the frequencies of the transmitted and reflected waves are the same as that of the incident wave, further verifying that the simulations were performed in the harmonic regime and that scattering was only due to the mass discontinuity at the interface.

This analysis also shows that some branches of the vibrational spectrum never participate in the scattering even when they contain modes that have the same frequency as the incident LA wave. For example, we find that the torsional acoustic modes are never excited in either the transmitted or reflected waves. We have also found that none of the degenerate branches, including the TA branches, are ever excited by the incident LA wave packet. In addition, certain nondegenerate branches do not participate in the scattering event. Based on these observations, it is clear that frequency considerations alone cannot explain the scattering process and that there must be general selection rules for the nanowire scattering studied here.

These selection rules can be understood using symmetry arguments. To develop this perspective, it is necessary to consider the symmetry group of the nanowire with and without the mass discontinuity. When there is no mass discontinuity, the space group symmetry of the nanowire is $D_{2 d}$. This space group has eight operations. In particular, four pointgroup operations comprise the point group $C_{2 v}$. The other four operations include lattice translations in addition to rotations and inversions. We expect that the eigenstates of the dynamical matrix should reflect the underlying point-group symmetry of the dynamical matrix. From the basic principles of group theory, the full space group $D_{2 d}$ therefore has one two-dimensional and four one-dimensional representations. ${ }^{26}$ Each eigenstate corresponds to a different representation of the symmetry, and hence each transforms differently when operated on by the elements of the group. The nanowire spectrum for the system without the mass discontinuity 


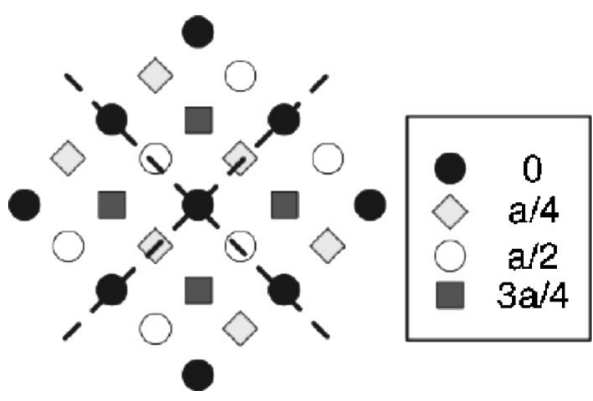

FIG. 6. Schematic of the mirror symmetry planes (represented by dashed lines) of the $C_{2 v}$ point group shown with the atomic positions for a cross section of one repeat unit of the $2 \times 2$ nanowire. The atomic planes are each represented by different symbols depending on their $z$ coordinate (with the $z$ axis along the nanowire axis) as indicated in the legend. In addition to the mirror planes, the group includes the identity and rotation by $\pi$ around the nanowire axis. The resulting group therefore has four distinct symmetry operations, and therefore four distinct representations (see Table I).

(shown in Fig. 2) has doubly degenerate branches (e.g., including the TA branches) and four different nondegenerate branches.

The mass discontinuity breaks the translational symmetry of the nanowire and, as a result, is not described by the $D_{2 d}$ space group. However, the mass discontinuity does not break the symmetry of the point group $C_{2 v}$. The elements of the point group $C_{2 v}$ include the identity, rotation by $\pi$ about the nanowire axis, and two mirror planes; they are shown schematically in Fig. 6. Because this group has four elements, we expect that the normal modes of the system with the interfaces of materials $A$ and $B$ should have four different nondegenerate representations. ${ }^{26}$ We show in Table I the character table of this group.

To understand the scattering, we have analyzed the character of each of the branches shown in Fig. 2 in terms of the group $C_{2 v}$. We do this by operating on the eigenvectors with the operations of the point group $C_{2 v}$, and then projecting the result back onto the original eigenvector. To determine the particular representation, we refer to the character table in Table I. For the nondegenerate modes in Fig. 2, we always find that the character corresponds to either the $A_{1^{-}}$or $A_{2}$-type mode from Table I. For example, the character of the LA branch corresponds to that of an $A_{1}$ mode: thus all of the incident phonon wave packets used in this study have $A_{1}$ symmetry. The torsional acoustic mode, on the other hand, is an $A_{2}$ mode. Because there is no two-dimensional representation of the group $C_{2 v}$, it may seem that the degenerate modes will not correspond to the characters in Table I. However, because the modes are degenerate in the nanowire with-

TABLE I. Character table for the point group $C_{2 v}$. The operations of the group include identity $E$, rotation by $\pi$ about the nanowire axis, and two mirror planes. These are shown schematically for a cross section of the nanowire in Fig. 6.

\begin{tabular}{llrrr}
\hline \hline & $E$ & $C_{2}$ & $\sigma_{v}$ & $\sigma_{v}^{\prime}$ \\
\hline$A_{1}$ & 1 & 1 & 1 & 1 \\
$A_{2}$ & 1 & 1 & -1 & -1 \\
$B_{1}$ & 1 & -1 & 1 & -1 \\
$B_{2}$ & 1 & -1 & -1 & 1 \\
\hline \hline
\end{tabular}
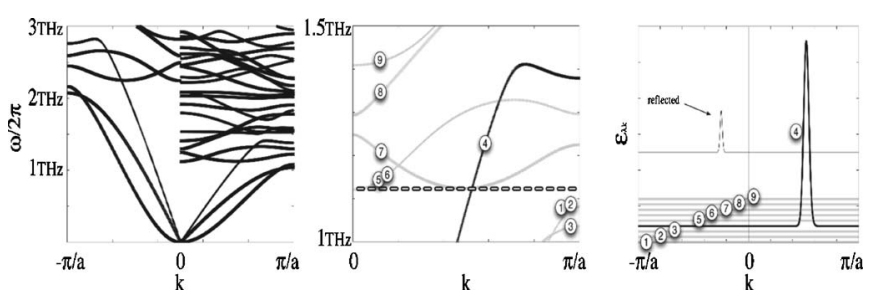

FIG. 7. In the left panel, the phonon dispersions are shown for modes in material $A$ (negative $k$ values) and for modes in material $B$ (positive $k$ values) for the $2 \times 2$ nanowire. The phonon is incident from the LA band in material $A$ and is transmitted into modes in material $B$. The center panel shows the modes in material $B$ that are relevant for the transmission of a $1.12 \mathrm{THz}$ mode incident from material $A$. The right panel shows the result of the analysis using Eqs. (2) and (3) for the energy contained in each mode after the scattering. The dark lines in the center and right panels are for $A_{1}$ branches, whereas the gray lines are the $A_{2}$ and degenerate branches. The reflected mode in material $A$ is in the LA branch, but now with negative $k$. The transmitted bands are labeled according to the labeling of the bands in the center panel. As can be seen, only band 4, which is the LA band in material $B$, transmits energy.

out the mass discontinuity, the polarization is arbitrary, and we can choose the polarization in any way we desire. We have in fact found that the polarization of the degenerate modes can be chosen in such a way that these modes have a character that corresponds exactly to the $B_{1}$ and $B_{2}$ representations of $C_{2 v}$. Because the $B_{1}$ and $B_{2}$ representations are not degenerate in $C_{2 v}$, this tells us that the interface breaks the degeneracy. The fact that the polarization of the degenerate modes is important for interfacial scattering is closely related to our previous study of scattering in bulk silicon, where it was found that the transmission coefficient $\alpha$ for TA modes depends strongly on the polarization of the incident wave, especially for shorter wavelengths. ${ }^{13}$

The representation of the normal modes is directly related to whether or not they participate in the scattering. In particular, we find that only $A_{1}$ modes are excited by the incident $A_{1}$-type LA wave packet. The $A_{2}$ modes and the degenerate modes, which are arbitrary mixtures of the $B_{1}$ and $B_{2}$ representations of the $C_{2 V}$ group, do not ever participate in the scattering of a LA mode. This point is demonstrated graphically in Fig. 7. Figure 7 shows the result of scattering due to an incident wave LA packet with a frequency of $1.12 \mathrm{THz}$. The left panel of Fig. 7 shows the phonon dispersions of materials $A$ and $B$. The center panel focuses in more closely on the relevant frequency range for transmission into material $B$, with the branches numbered. Finally, the right panel of Fig. 7 shows the energy transmitted into the various modes, with each contribution labeled to refer to labeled bands in the center panel. As can be seen from the right panel of Fig. 7, only band 4 in material $B$ is transmitting energy. The three other bands that have modes with a frequency of $1.12 \mathrm{THz}$ do not transmit any energy. For example, the degenerate branches labeled 5 and 6 do not transmit energy. Also, band 7, which we have identified as being an $A_{2}$-type mode, does not transmit energy.

Another typical case is shown in Fig. 8 for the scattering of an incident LA wave packet with an average frequency of $2.10 \mathrm{THz}$ in the $4 \times 4$ nanowire. The center panel of Fig. 8 shows that only one $A_{1}$ branch (labeled as branch 35 in Fig. 8) has the appropriate frequency to transmit energy into ma- 


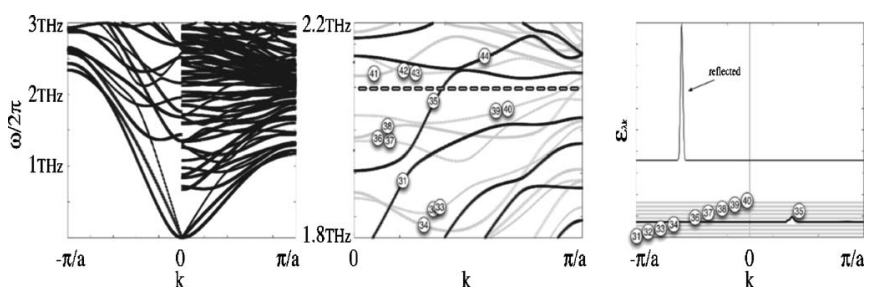

FIG. 8. In the left panel, the phonon dispersions are shown for modes in material $A$ (negative $k$ values) and for modes in material $B$ (positive $k$ values) for the $4 \times 4$ nanowire. The center panel shows the modes in material $B$ that are relevant for the transmission of a $2.10 \mathrm{THz}$ mode incident from material $A$. The right panel shows the result of the analysis using Eqs. (2) and (3) for the energy contained in each mode after the scattering. The dark lines in the center and right panels are for $A_{1}$ branches, whereas the gray lines are the $A_{2}$ and degenerate branches. The transmitted bands are labeled according to the labeling of the bands in the center panel. As can be seen, only band 35 , which is an $A_{1}$ mode in material $B$, transmits energy.

terial $B$. There are other branches that have the right frequency but are not the appropriate representations to transmit the energy. In particular, branches 38, 39, and 40 have frequencies that match the frequency of the incident LA mode. Branch 38 is an $A_{2}$ mode, and branches 39 and 40 are degenerate modes and hence are combinations of $B_{1^{-}}$and $B_{2}$-type modes. The analysis of the transmitted energy in the right panel of Fig. 8 clearly shows that only branch 35 , the $A_{1}$ mode, transmits any energy.

The results of this analysis fully verify that the scattering at the mass discontinuity in the nanowire does not mix the various representations of the $C_{2 v}$ point group. This conclusion is not surprising. The normal modes of the nanowire with the mass discontinuity can be constructed by mixing the normal modes of a wire composed entirely of material $A$ and another wire made from material $B$. For our group theoretical arguments, we know how the modes of the nanowire with the mass discontinuity should behave when operated on by the elements of the $C_{2 v}$ (see the character table in Table I). It is clearly only possible to make an $A_{1}$ mode if we mix $A_{1}$ modes from materials $A$ and $B$. For the scattering problem, this is analogous to the observation that an incident LA mode which is an $A_{1}$ mode only scatters into other $A_{1}$ modes. The same argument applies to the other three representations $A_{2}$, $B_{1}$, and $B_{2}$.

In Fig. 9 we show the phonon dispersions of the nanowire separately for the $A_{1}, A_{2}$, and degenerate bands for the $2 \times 2$ nanowire. The left portion (negative $k$ values) of each panel shows the phonon dispersions for material $A$ where the incident phonon is created, while the left portion of each panel (positive $k$ values) shows the modes in material $B$, and hence represent the available states for transmission. The complete phonon dispersion for the $2 \times 2$ nanowire, which is the combination of these three distinct types, is shown in Fig.
2 for material $A$. The analysis described above has in all cases shown that only $A_{1}$-type modes in the left panel of Fig. 9 are required to describe the scattering of $A_{1}$ LA modes from the interface. By contrast, none of the bands shown in the center and right panels of Fig. 9, which correspond to the $A_{2}$ and degenerate (i.e., $B_{1}$ and $B_{2}$ ) modes respectively are ever involved in the scattering. It is interesting to note that there are two small regions in the left panel of Fig. 9 for material $B$ where there are gaps (i.e., a range of frequency where there are no $A_{1}$-type modes). These gaps occur near 145 and $2.65 \mathrm{THz}$ in material $B$. The frequencies do in fact correspond to minima in the transmission coefficient, as we expected (see Fig. 5). While we might expect the transmission coefficient to be zero in these gap regions, the fact that the wave packet has a finite extent means that the wave packet is characterized by a range of frequencies which in this case is always bigger than the gaps in the spectrum. In particular, the gap near $1.45 \mathrm{THz}$ is about $0.06 \mathrm{THz}$ in width, while the gap near $2.65 \mathrm{THz}$ is about $0.08 \mathrm{THz}$ wide. The wave packets themselves span a range of frequencies in these regimes of about $0.17 \mathrm{THz}$, which is significantly bigger than the width of the two gap regions, and hence we should not expect a complete reflection of the incident wave packet. If the incident wave packet had been more delocalized in real space, the spread of incident frequencies would correspondingly be smaller, and it should be possible to observe a complete reflection at these gaps. These observations also suggest that the particular values of the transmission coefficient depend somewhat on the size of the incident wave packet. In particular, the results we obtain are expected to depend somewhat on the range of wave numbers used to generate the localized wave packet.

Having identified that the interface scattering does not couple different representations, it is useful to consider the DOS for each representation separately to identify where accessible states for phonon transmission exist and where there are gaps in the spectrum. In Fig. 10 we show the DOS due to the $A_{1}, A_{2}$, and degenerate modes separately for the $2 \times 2$ nanowire made of material $B$. The total DOS for material $B$ is also shown for comparison. The total DOS, while there is a significant structure resulting from the small diameter of the nanowire, is essentially a continuous spectrum, with only a small gap (about $0.06 \mathrm{THz}$ in width) appearing near 4.0 THz. However, the DOS due to the $A_{1}, A_{2}$, and degenerate modes show gaps over the entire frequency range. For example, the DOS of the $A_{1}$ modes has small gaps at 1.45 and $2.65 \mathrm{THz}$, as previously noted. Looking further across the spectrum, there are also gaps near 4.0 and $7.8 \mathrm{THz}$. The $A_{2}$ DOS has many more gaps. Furthermore, the gaps are much wider than those found in the spectrum of the $A_{1}$

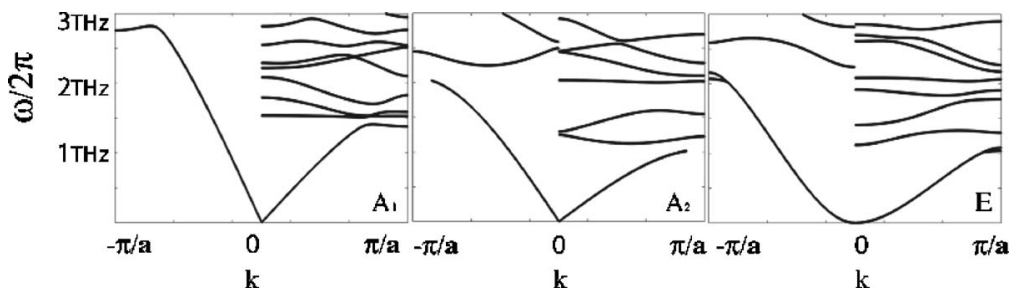

FIG. 9. Phonon dispersions separated by representation for the $2 \times 2$ nanowire. The left part of the spectrum is for material $A$, while the right part of the spectrum is shifted down in frequency by a factor of 2 and hence represents material $B$. Phonons incident in the LA branch in material $A$ are transmitted only into these $A_{1}$ modes in material $B$. 


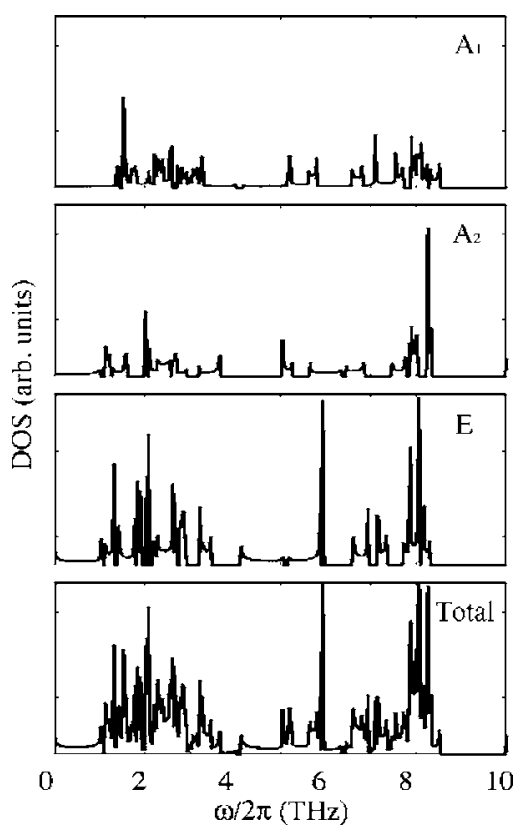

FIG. 10. DOS for the $A_{1}, A_{2}$, and degenerate modes in material $B$ for the $2 \times 2$ nanowire structure. For comparison, the total DOS is also included. In spite of showing a significant structure, the total DOS does not exhibit gaps. However, the spectra due to the different representations do show gaps.

modes. For example, a rather broad gap appears in the $A_{2}$ spectrum from about 3.68 to $5.02 \mathrm{THz}$. Another wide gap appears between 6.88 and $7.44 \mathrm{THz}$. The DOS due to the degenerate modes also has wide gaps in the spectrum. Most notably, there are extremely wide gaps between 3.48 and $4.10 \mathrm{THz}$ and 5.98 and $6.58 \mathrm{THz}$. A possible explanation for the wider gaps in the $A_{2}$ and degenerate modes DOS is that these mode consist at least partly of transverse and torsional type modes which, compared to the longitudinal modes that partly characterize the $A_{1}$ modes, have relatively low group velocities and hence sharper and more discrete features in the DOS. Independent of the reasons for these differences, it is clear that the discrete nature of the $A_{2}$ and degenerate-mode spectrum indicates that in general we should expect an even lower transmission at the interface when a phonon from these branches is incident on the interface, including significant portions of the spectrum that will be completely reflected.

The presence of minima and gaps in the DOS is strongly a function of the size of the system. In particular, as the nanowire diameter increases, the DOS becomes less discrete and gaps and minima less pronounced. For large enough nanowires, the gaps in the spectra should disappear entirely. To demonstrate this point, we show in Fig. 11 the DOS for the $4 \times 4$ nanowire. For the $A_{1}$-type modes, there are in fact no actual gaps in the spectrum. However, there are several minima which are found to correspond to minima in the energy transmission coefficient $\alpha$. For example, the scattering analysis in Fig. 8 for the $4 \times 4$ nanowire for an incident LA wave packet with a frequency of $2.10 \mathrm{THz}$ shows that very little energy is transmitted across the interface. In particular, the energy transmission coefficient $\alpha$ at this frequency is 0.04 . An inspection of the DOS for the $A_{1}$ modes in Fig. 11 at this frequency indicates a minimum at this fre-

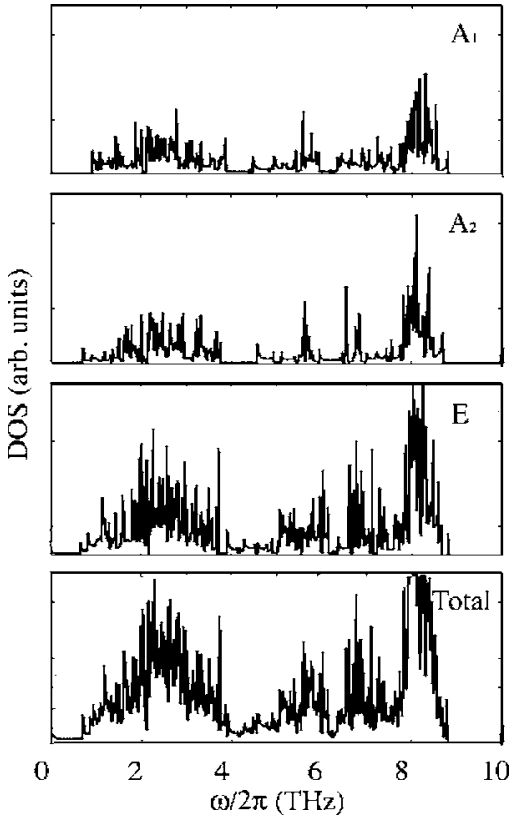

FIG. 11. DOS for the $A_{1}, A_{2}$, and degenerate modes in material $B$ for the $4 \times 4$ nanowire structure. For comparison, the total DOS is also included. While the DOS shows the presence of gaps and minima, a comparison to Fig. 10 shows that some gaps have disappeared or are narrowed as a result of the larger diameter of the nanowire.

quency, although not actually a gap. This suggests that even when there are no actual gaps in the spectra, minima in the DOS which will persist to large-diameter nanowires are correlated with the low energy transmission across the interface. For the $A_{2}$ and $E$ modes, there are still gaps present in the spectrum, indicating that there will be regimes of complete reflection for incident modes of these types. However, a comparison of Figs. 10 and 11 shows that the gaps are considerably narrower in the $4 \times 4$ nanowire than in the $2 \times 2$ nanowire, as we should expect. Some gaps present in the 2 $\times 2$ nanowire are not present at all in the larger diameter 4 $\times 4$ nanowire.

Finally, we observe that for some frequencies, the transmission coefficient is actually larger than the value $8 / 9$ predicted by the AMM. In the low-frequency regime, most of the energy is transmitted into the LA modes in material $B$. For the $2 \times 2$ wire, the LA branch turns over near the zone edge. This allows for more than one possible $k$ value for transmission, and hence results in the gradual increase in $\alpha$ above $8 / 9$. For the $4 \times 4$ and $6 \times 6$ wires, the optical branches of the $A_{1}$ type are present at lower frequencies, and some energy is transmitted into these branches. The energy transmitted in the optical branches in the low-frequency regime is fairly small, but is enough to increase the energy transmission above the value of $8 / 9$ predicted by the AMM.

\section{CONCLUSIONS}

We have shown that wave packet dynamics can be used to understand fundamental scattering processes at an interface in a nanowire. While this particular interfacial system cannot be realized experimentally, it is representative of a wide range of semiconductor nanostructures. Consequently, the results obtained are rather general and can lead to insight 
into, for example, $\mathrm{Si} / \mathrm{Ge}$ superlattice nanowires, where the $\mathrm{Si} / \mathrm{Ge}$ layers are epitaxial and the primary scattering comes from differences in mass and bonding.

We have shown how the selection rules for transmission can be understood from a simple analysis of the underlying symmetry of the nanowire. This suggests that the energy transmission coefficient depends not only on the diameter of the nanowire, but also rather significantly on the underlying symmetry (i.e., the shape) of the nanowire structure.

This observation suggests interesting possibilities for phonon transmission in real nanowires. For example, $\mathrm{Si} / \mathrm{Ge}$ superlattice nanowires have been studied for applications in thermoelectrics. Because epitaxial interfaces can be constructed, it should be possible to realize a high-symmetry nanowire like the one studied here. Indeed, synthesis techniques have advanced to the stage where extremely thin nanowires can be fabricated with a variety of growth axes, including $\langle 110\rangle,{ }^{27,28}\langle 100\rangle,{ }^{27,29}\langle 111\rangle,{ }^{28}$ and $\langle 112\rangle .{ }^{28}$ In a real system, there will be point defects and surface roughness, and transport will not be in a completely ballistic regime that our study addresses. Defects will certainly result in a structure of lower symmetry, and as a result the selection rules identified here can only be considered approximate. At this time it is difficult to say with certainty whether a wire of lower symmetry will result generally in lower or higher energy transmission. However, because the presence of high symmetry tends to close entirely some channels for transmission, this suggests that a wire with less symmetry, realized perhaps by changing the shape, will possibly be more efficient at transmitting phonons.

Another important consideration not taken into account here is the deformation at the interface. A lattice strain will exist at a real interface between two materials such as $\mathrm{Si}$ and Ge. While this might change the details of the scattering, however, the deformation is not expected to change the symmetry, and it is likely that the fundamental phenomena we suggest here should still be present. Without doing a detailed study, it is difficult to predict exactly how the results may depend on the interfacial strain.

The usefulness of a nanowire in applications depends on its thermal conductivity at its operating temperature. However, it is important to consider what experiments might be able to probe in detail the symmetry-dependent transport properties suggested here. One possibility is to use coherentphonon packets that can be produced by laser excitations. However, state-of-the-art coherent phonon pulses are limited to a maximum frequency of about $1 \mathrm{THz}$, and hence too low to probe the effects we predict. ${ }^{30}$ Another more promising approach is to measure the conductance at low temperatures, where higher-frequency phonons are frozen. This has been done for very low temperatures to observe the fundamental quanta of thermal conductance. ${ }^{31}$ At somewhat higher temperatures, experiments might be able to probe the detailed phonon transmission structures we observe, especially if the system is in the ballistic limit and relatively free of defects. From Fig. 5, it is clear that interesting effects are observed beginning at frequencies between 1 and $2 \mathrm{THz}$. This suggests that experiments should look in a temperature range beginning near $50 \mathrm{~K}$, at least for nanowires with diameters comparable to the ones studied here. A detailed experimental study of the low-temperature thermal conductance of superlattice nanowires with distinctly different shapes would be a very useful test of our results.

It is also instructive to consider our results in terms of the widely used and quite successful diffuse-mismatch model (DMM) for interfacial scattering. ${ }^{25}$ The DMM has been successful in predicting the Kapitza conductance of a variety of interfaces between different materials. In the DMM picture, scattering is elastic, but the final state excited by the incident phonon is chosen at random. As a result, the energy transmission coefficient is essentially determined by the relative number of modes present on either side of the interface. The results found here suggest not surprisingly that the DMM should not apply to a system free of defects. In particular, the high symmetry of the interface results in very directed scattering, with some possible channels being completely closed by symmetry considerations. However, it is still interesting to note that, above the cutoff for LA $\rightarrow$ LA transmission, while $\alpha$ depends strongly on the incident frequency, overall it is consistent with approximately $\alpha \sim 0.5$. This is approximately what the DMM would predict, although in this case it is clear that the agreement here is accidental. This observation may have important practical implications. In particular, it may be that even pristine interfaces between nanowires result in a rather poor energy transmission and a high Kapitza resistance, and attempts to increase the thermal conductivity through structural refinements may not be successful. In fact, because the selection rules identified here make some channels inaccessible for transmission, it may be that interfaces with defects, and hence a lower symmetry, actually transmit phonons more efficiently than perfect, highsymmetry interfaces.

It is important to note that these gap regions exist for a single interface and are distinct from gap states that are caused by band folding in a superlattice. Although our simulation cell did have periodic boundary conditions along the propagation direction, and hence in effect is a superlattice, the study applies to scattering from a single interface because we did not allow the system to evolve in time long enough for interference between different interfacial scattering events to occur. The transport through a superlattice will involve additional gaps introduced into the spectrum by interference effects, and the phonon transport through the superlattice would be even more difficult, leading to an even lower thermal conductivity. However, our results shed some light on why the nanowire transport, with or without a superlattice structure, can be quite low even with pristine surfaces and interfaces.

${ }^{1}$ T. G. Kim, X.-L. Wang, Y. Suzuki, K. Komori, and M. Ogura, IEEE J. Sel. Top. Quantum Electron. 6, 511 (2000).

${ }^{2}$ D. Yang and J. B. Khurgin, Superlattices Microstruct. 27, 245 (2000).

${ }^{3}$ A. Khitun, A. Balandin, K. L. Wang, and G. Chen, Physica E (Amsterdam) 8, 13 (2000).

${ }^{4}$ D. Li, Y. Wu, R. Fan, P. Yang, and A. Majumdar, Appl. Phys. Lett. 83, 3186 (2003).

${ }^{5}$ D. Li, Y. Wu, P. Kim, L. Shi, P. Yang, and A. Majumdar, Appl. Phys. Lett. 83, 2934 (2003).

${ }^{6}$ A. Majumdar, Science 303, 777 (2004).

${ }^{7}$ D. G. Cahill, W. K. Ford, K. E. Goodson, G. D. Mahan, A. Majumdar, H. 
J. Maris, R. Merlin, and S. R. Phillpot, J. Appl. Phys. 93, 793 (2003).

${ }^{8}$ N. Mingo, L. Yang, D. Li, and A. Majumdar, Nano Lett. 3, 1713 (2003).

${ }^{9}$ S. G. Volz and G. Chen, Appl. Phys. Lett. 75, 2056 (1999).

${ }^{10}$ C. Dames and G. Chen, J. Appl. Phys. 95, 682 (2004).

${ }^{11}$ J. Zou and A. Balandin, J. Appl. Phys. 89, 2932 (2001).

${ }^{12}$ Y. Chen, D. Li, J. Yang, Y. Wu, J. R. Lukes, and A. Majumdar, Physica B 349, 270 (2004).

${ }^{13}$ P. K. Schelling, S. R. Phillpot, and P. Keblinski, Appl. Phys. Lett. 80, 2484 (2002).

${ }^{14}$ H. Zhao and J. B. Freund, J. Appl. Phys. 97, 024903 (2005).

${ }^{15}$ P. K. Schelling and S. R. Phillpot, J. Appl. Phys. 93, 5377 (2003).

${ }^{16}$ P. K. Schelling, S. R. Phillpot, and P. Keblinski, J. Appl. Phys. 95, 6082 (2004).

${ }^{17}$ S. Sinha, P. K. Schelling, S. R. Phillpot, and K. E. Goodson, J. Appl. Phys. 97, 023702 (2005).

${ }^{18}$ F. F. Abraham and I. P. Batra, Surf. Sci. 163, L752 (1985).

${ }^{19}$ R. J. Hamers, R. M. Tromp, and J. E. Demuth, Phys. Rev. B 34, 5343 (1986).

${ }^{20}$ F. H. Stillinger and T. A. Weber, Phys. Rev. B 31, 5262 (1984).
${ }^{21}$ E. G. Keim, J. Wormeester, and A. van Silfhout, J. Vac. Sci. Technol. A 8, 2747 (1990).

${ }^{22}$ P. K. Schelling and P. Keblinski, Phys. Rev. B 68, 035425 (2003).

${ }^{23}$ T. Thornhauser and G. D. Mahan, Phys. Rev. B 69, 075213 (2004).

${ }^{24}$ N. Mingo, Phys. Rev. B 68, 113308 (2003).

${ }^{25}$ E. T. Swartz and R. O. Pohl, Rev. Mod. Phys. 61, 605 (1989).

${ }^{26} \mathrm{M}$. Tinkham, Group Theory and Quantum Mechanics (McGraw-Hill, New York, 1964).

${ }^{27}$ J. D. Holmes, K. P. Johnston, R. C. Doty, and B. A. Korgel, Science 287, 1471 (2000).

${ }^{28}$ Y. Wu, Y. Cui, L. Huynh, C. J. Barrelet, D. C. Bell, and C. M. Lieber, Nano Lett. 4, 433 (2004).

${ }^{29}$ D. D. D. Ma, C. S. Lee, F. C. K. Au, S. Y. Tong, and S. T. Lee, Science 299, 1874 (2003).

${ }^{30}$ D. G. Cahill, W. K. Ford, K. E. Goodson, G. D. Mahan, A. Majumdar, H. J. Maris, R. Merlin, and S. R. Phillpot, J. Appl. Phys. 93, 793 (2003).

${ }^{31}$ W. Fon, K. C. Schwab, J. M. Worlock, and M. L. Roukes, Phys. Rev. B 66, 045302 (2002). 